\title{
The impact of occipital lobe cortical thickness on cognitive task performance: an investigation in Huntington's Disease
}

Eileanoir B Johnson ${ }^{1}$, Elin M Rees ${ }^{1}$, Izelle Labuschagne ${ }^{2,3}$, Alexandra Durr ${ }^{4}$, Blair R Leavitt ${ }^{5}$, Raymund A C Roos ${ }^{6}$, Ralf Reilmann ${ }^{7}$, Hans Johnson ${ }^{8}$, Nicola Z Hobbs ${ }^{1}$, Douglas R Langbehn ${ }^{9}$, Julie C Stout ${ }^{2}$, Sarah J Tabrizi ${ }^{1}$, Rachael I Scahill ${ }^{1}$ and the TRACK-HD investigators

1. UCL Institute of Neurology, University College London, UK.

2. School of Psychological Sciences, Monash University, Clayton, Victoria, Australia.

3. School of Psychology, Australian Catholic University, Melbourne, Victoria, Australia.

4. Department of Genetics and Cytogenetics, and INSERMUMR S679, APHP, ICM Institute, Hôpital de la Salpêtrière, Paris, France.

5. Centre for Molecular Medicine and Therapeutics, Department of Medical Genetics, University of British Columbia, Vancouver, BC, Canada.

6. Department of Neurology, Leiden University Medical Centre, Leiden, Netherlands.

7. George-Huntington-Institute, Germany.

8. Department of Psychiatry, University of lowa, Iowa City, IA, USA.

9. Department of Biostatistics, University of lowa, lowa City, IA, USA. 
Running head: OCCIPITAL CORTEX IN HD

For correspondence:

Dr Rachael Scahill

Huntington's Disease Research Group

UCL Institute of Neurology

Queen Square

London

WC1N 3BG

Email: $\underline{\text { r.scahill@ucl.ac.uk }}$

Tel: +44 2031087477 


\section{ABSTRACT}

Background: The occipital lobe is an important visual processing region of the brain. Following consistent findings of early neural changes in the occipital lobe in Huntington's disease (HD), we examined cortical thickness across four occipital regions in premanifest (preHD) and early HD groups compared with controls. Associations between cortical thickness in gene positive individuals and performance on six cognitive tasks, each with a visual component, were examined. In addition, the association between cortical thickness in gene positive participants and one nonvisual motor task was also examined for comparison.

Methods: Cortical thickness was determined using FreeSurfer on T1-weighted 3T MR datasets from controls $(\mathrm{N}=97)$, preHD $(\mathrm{N}=109)$ and $\mathrm{HD}(\mathrm{N}=69)$ from the TRACK-HD study. Regression models were fitted to assess between-group differences in cortical thickness, and relationships between performance on cognitive tasks, the motor task and occipital thickness was examined in a subset of genepositive participants $(\mathrm{N}=141)$.

Results: Thickness of the occipital cortex in preHD and early HD participants was reduced compared with controls. Regionally-specific associations between reduced cortical thickness and poorer performance were found for five of the six cognitive tasks, with the strongest associations in lateral occipital and lingual regions. No associations were found with the cuneus. The non-visual motor task was not associated with thickness of any region.

Conclusions: The heterogeneous pattern of associations found in the present study suggests that occipital thickness negatively impacts cognition, but only in regions that are linked to relatively advanced visual processing (e.g., lateral occipital, lingual regions), rather than in basic visual processing regions such as the cuneus. 
Running head: OCCIPITAL CORTEX IN HD

Our results show, for the first time, the functional implications of occipital atrophy highlighted in recent studies in HD.

Keywords: FreeSurfer, Huntington's Disease, MRI, Occipital lobe, Visual cortex 
Running head: OCCIPITAL CORTEX IN HD

\section{Introduction}

The occipital cortex is primarily viewed as the visual area of the brain, with topologically-distinct regions believed to manage different aspects of visual processing (Wandell et al., 2007). In Huntington's Disease (HD), a progressive genetic neurodegenerative disease, atrophy in the occipital cortex has been identified as an early and prominent element of neurodegeneration, although this atrophy in the occipital lobe has not been the focus of research to date (Rosas et al., 2002; Tabrizi et al., 2012). The regional specificity of occipital changes in HD is unknown, as are the functional implications of occipital cortex thinning on cognition. As most cognitive tasks involve a visual component, for example written responses or symbol identification, impaired visual processing due to occipital cortex pathology could manifest as poorer performance on these tasks. This study used an HD sample to describe the regional pattern of cortical thinning in the occipital cortex, across the spectrum from premanifest (preHD) to early HD, and the associations between occipital atrophy and performance on cognitive tests involving a visual component.

The visual system is a complex integrative system, with a ventral steam thought to process colour and discriminate shapes and objects, and a dorsal stream that processes spatial information, motion and visually-guided grasping and reaching (Hebart and Hesselmann, 2012). It has long been thought that specific regions of the occipital cortex are associated with different visual processing functions that make up these two streams (Hebart and Hesselmann, 2012). Consequently, the location of occipital cortex pathology is likely to affect the manifestation of any visual deficit. Current understanding of the functional topology of the occipital cortex, which is derived from visual field mapping and supported by functional imaging studies (see Cabeza and Nyberg (2000)), divides the occipital cortex into a number of processing areas that extend over the occipital gyri. Briefly, the primary visual area (V1), also called Brodmann Area (BA) 17, is located within the pericalcarine region and maps the signals received from the retina in a topographic manner (Tootell et al., 1998; Wandell et al., 2007). This visual information is also projected to higher-level processing regions (Roe and Ts'o, 1995), known as V2, V3 and V4. Visual area 2 (V2, BA18) is spread across part of the 
Running head: OCCIPITAL CORTEX IN HD

cuneus above $\mathrm{V} 1$, and the lingual gyrus below $\mathrm{V} 1 . \mathrm{V} 2$ is thought to process basic visual characteristics such as colour and orientation (Roe and Ts'o, 1995; Zeki, 1978). Visual area 3 (V3), believed to play a role in motion perception (Gegenfurtner et al., 1997; Larsson and Heeger, 2006), is also located within the cuneus but extends laterally. The lateral occipital cortex (LOC) can refer to a small sub-region of the lateral side of the occipital cortex, but for the current study it refers to the entire lateral side of the occipital cortex. This region is thought to integrate visual information, especially shape information, along with visual and tactile object recognition (Beauchamp, 2005; Grill-Spector et al., 2001; Larsson and Heeger, 2006). The LOC also plays a role in tasks involving the performance of motor actions and tactile stimulation, especially movements involving the limbs (Amedi, 2002; Astafiev et al., 2004). Within the lingual gyrus is visual area 4 (V4), a colour processing area also thought to identify words and letters (Mechelli et al., 2000; Tootell et al., 2003). To summarise, regions of the occipital cortex, (described here in relation to the pericalcarine, cuneus, lingual and LOC) perform topologically-specific functions in visual processing. It is currently unclear what effect pathology within these regions has on performance levels for cognitive tasks with visual components.

HD has an onset in mid-adulthood and is characterised by progressive motor, cognitive and psychiatric symptoms. There is a long premanifest period (referred to as preHD) during which subtle signs of the emerging disease are detectable (Paulsen et al., 2008; Tabrizi et al., 2009). The neuropathology of HD is primarily focused within the striatum of the basal ganglia, although extra-striatal and cortical regions are also affected (Tabrizi et al., 2012; Vonsattel and DiFiglia, 1998). Brain atrophy has been shown to begin many years before disease onset and progresses from preHD to manifest disease with increasing disease burden (Tabrizi et al., 2011, 2009). Several imaging studies have detected occipital cortex atrophy in both pre- and manifest HD (Rosas et al., 2008; Stoffers et al., 2010; Tabrizi et al., 2011, 2009; Wolf et al., 2014) and there is a marked reduction in number of neurons in the occipital cortex in HD (Lange, 1981; Rüb et al., 2015). In addition, performance deficits 
Running head: OCCIPITAL CORTEX IN HD

on cognitive tasks involving a visual component have been widely reported in both preHD and early HD (Paulsen et al., 2014; Tabrizi et al., 2013) and a small number of studies have identified difficulties with visual processing, although this is not reported as a common feature of the disease. Early HD participants suffer from motion discrimination deficits, but not deficits in static contrast sensitivity, and preHD participants generally show no deficit in these tasks (O'Donnell et al., 2008, 2003). An HD cohort ranging from pre- to manifest disease would therefore be expected to exhibit varying levels of both cognitive impairment and occipital cortical thickness, with some possible deficits in motion processing.

In addition, previous studies have also found significant relationships between cognitive performance and brain volume in HD. Occipital grey matter volume was found to be significantly related to time to complete a visual discrimination task (Gomez-Anson et al., 2009), and emotion recognition (Scahill et al., 2013). Cortical thickness of the occipital region has been associated with performance on the Symbol Digit task and a Stroop Colour Reading task, although these analyses were whole-brain studies with no correction for multiple comparisons (Rosas et al., 2008, 2005). These studies suggest that there is a relationship between occipital lobe thickness and cognitive performance in $\mathrm{HD}$, however a regional analysis allowing for in-depth characterization of the specific relationship between sub-regions of the occipital lobe and cognitive performance across varying disease stages has not yet been performed. Furthermore, without controlling for other factors likely to contribute to cognitive performance, such as disease progression and education, it is unclear whether the occipital lobe uniquely contributes to cognitive performance.

This study aimed to test unique associations between thickness of the visual cortex and cognitive test performance in HD. Cortical thickness in four occipital regions (pericalcarine, cuneus, lingual and LOC) was compared between healthy controls, preHD and manifest HD participants, and the relationship between occipital cortex thickness and cognitive impairment was examined in the HD gene-carriers. It was hypothesized that the thickness of the cuneus, lingual region and the LOC, which are areas known to be involved 
Running head: OCCIPITAL CORTEX IN HD

in functions such as motion detection, visual memory, integration of visual information and object recognition, would be associated with cognitive test performance. In contrast, as visual field deficits are not known to occur in HD, it was hypothesized that that the pericalcarine (V1) would not show such associations.

\section{Materials and Methods}

\subsection{Participants}

Participants were recruited across four study sites as part of the TRACK-HD study (Tabrizi et al., 2009), 313 participants from the 2010 time-point were included in the present investigation of the occipital cortex (107 controls, 116 preHD and $90 \mathrm{HD}$ individuals). The preHD cohort was separated into two groups based on the median expected years to disease onset; those estimated to be more than 10.8 years from disease onset (Langbehn et al., 2010) were classified as the preHD-A group and those less than 10.8 years from estimated onset, preHD-B. Using the Unified Huntington's Disease Rating Scale (UHDRS;(Huntington Study Group, 1996)) the HD cohort was classified based on their Total Functional Capacity (TFC) scores as HD1 (TFC=11-13) or HD2 (TFC=7-10); HD2 being the more advanced group. The control group was comprised of partners, spouses and genenegative siblings of the gene-carriers. Full selection criteria and data collection processes have been published previously (Tabrizi et al., 2009).

Participants were tested for visual function using a Snellen Visual Acuity equivalent, the Low-Contrast SLOAN Letter Charts (Balcer et al., 2000). This provided a score ranging from 1-12, with 1 representing poor visual acuity (20/200 vision) and 12 representing high acuity (20/16 vision). Participants with a score of less than 11 , which is equivalent to below the average 20/20 vision, were excluded from the current study. The remaining cohort for group cortical thickness comparisons comprised 275 participants (97 healthy controls, 51 preHD-A, 58 preHD-B, 40 HD1 and 29 HD2 participants). A subgroup of gene-positive participants was then used to relate cognitive performance to occipital lobe thickness. Participants who had manually measured caudate volumes already available and a score on 
Running head: OCCIPITAL CORTEX IN HD

the Hospital Anxiety and Depression Scale (HADS) were included in this analysis. This group consisted of 141 gene-positive participants; 36 preHD-A, 44 preHD-B, 37 HD1 and 24 HD2 participants. Participants with missing data for a cognitive test were excluded from the analysis for that individual test. Ethical approval was given by the local ethical committees and written informed consent was obtained from each participant according to the Declaration of Helsinki.

\subsection{Clinical Assessments}

A selection of cognitive tests with a large visual component and relatively low cognitive demand was selected from the larger TRACK-HD study battery (Tabrizi et al., 2009). The Paced Tapping task, a motor task without a visual component, was also included as a general proxy for disease progression to test the exclusivity of the visual-occipital relationship (Tabrizi et al., 2013).

1. Symbol Digit Modalities Test, SDMT (Smith, 1991). The SDMT is a pencil-andpaper task that assesses visuomotor integration and has components of visual scanning and tracking. Participants view a page that has a key at the top showing symbols with the digits 1-9. On the rest of the page, symbols are presented with empty boxes below, and participants are given 90 seconds to write in the empty boxes the digits paired with the symbols. The final score is the number of correctly matched symbols.

2. Stroop Word Reading (MacLeod, 1991). This subset of the Stroop task requires visual scanning, cognitive control and processing speed. Participants must read as many names of colours from a presented card as possible in 45 seconds and are scored by the number of words correctly read out loud.

3. Trails A (Spreen and Strauss, 1985). The Trail Making Task measures sustained attention and information processing speed. Participants are timed whilst drawing an ordinal line through randomly dispersed numbers from 1 to 25. The final score is the time taken to complete the task. 
4. Map Search (Robertson et al., 1994). This is a test of visuospatial selective attention. Participants search a visually-cluttered display to identify target symbols amongst distractors and are scored by the total number of symbols identified in two minutes.

5. Mental Rotation (Shepard and Metzler, 1971). The mental rotation task assesses spatial processing. Participants are asked to compare two figures and determine whether they are identical but rotated, or mirror image figures. Four conditions are included to assess rotations of different difficulty levels from easy (5 degrees) to difficult (305 degrees). There are many outcome measures available for this task; in the current study total score was percentage of items answered correctly.

6. Spot the Change (Cowan et al., 2005). This test examines the participant's ability to sustain object and location representations without rehearsal (visual working memory). An array of 5 coloured squares is briefly presented for $250 \mathrm{~ms}$. This is followed by an identical array, where one of the squares is encircled. The participant has to decide whether that square has changed colour and select from the responses 'same' or 'different'. The total score is the percentage of trials correct.

7. Paced Tapping (Reilmann et al., 2005). The Paced Tapping task is a measure of motor performance and control, and does not have a visual component. Participants are required to hold a computer mouse and tap alternating fingers at the same speed as a previously played $3.0 \mathrm{~Hz}$ tone. The outcome measure is deviation in tapping pace compared to the tone. Paced Tapping is a significant predictor of disease progression (Tabrizi et al., 2013).

In order to exclude any effect of depressive symptoms on cognitive and motor task performance, the HADS was used to score participants on depression (Zigmond and Snaith, 1983). The HADS is a self-rating scale with 14 items, each item scored from 0-3. Seven of the questions assess anxiety and 7 assess depression. Only the 7 depression questions 
Running head: OCCIPITAL CORTEX IN HD

were included in the current study, resulting in a score of 0-21 with 21 representing higher levels of depression. The HADS has previously been found to be a good screening measure for depression in HD (De Souza et al., 2010).

\subsection{Image Acquisition}

3T T1-weighted scans were acquired from four scanners using acquisition protocols previously validated for multi-site use (Tabrizi et al., 2009). T1-weighted scans were bias corrected using the non-parametric non-uniform intensity normalization (N3) method (Sled et al., 1998), with optimised parameters for 3T data as outlined in Boyes et al. (2008). All images were visually assessed for quality; specifically artefacts such as motion, distortion and poor tissue contrast (IXICO Ltd. and TRACK-HD imaging team, London, UK).

\subsection{Image Analysis}

The analysis was run using the default FreeSurfer version 5.3.0 pipeline with the -3T flag to optimise analysis for 3T data (Fischl and Dale, 2000; Fischl et al., 2004a, 2002). Trained analysts visually inspected segmentation outputs. No participants were excluded from the analysis due to segmentation failures.

The cortical thickness analysis was limited to the four occipital regions defined by the Desikan-Killiany atlas (Desikan et al., 2006): the cuneus, pericalcarine, lingual and LOC (shown in Figure 1). Two separate image analyses were conducted. In the first analysis, average thickness values for the four regions were initially extracted from each scan. Then, a weighted sum of these extracted mean thickness values was calculated to estimate the average occipital cortex. The weight for each region was computed by dividing the surface area of that region by the total surface area as: Average thickness of region $A$ and $B=$ $(($ Thickness region $A *$ Surface area region $A)+($ Thickness region $B *$ Surface area region $B)) /$ (Surface area region $\mathrm{A}+$ Surface area region $\mathrm{B}$ ). This calculation accounts for different surface areas of the four regions. After comparing thickness between right and left hemispheres and finding no significant difference for any region, thickness was averaged 
across the two hemispheres for each region using the same calculation as above to account for differences in surface area.

In the second analysis, statistical maps of the between-group differences and the clinical associations were processed in FreeSurfer in order to visualise the results. For this, data from all participants were pooled to create a study-specific group template by resampling each participant's data into a common space, performing spatial smoothing, and then combining all scans into a single file. Average thickness data from all participants were overlaid on this template and smoothed with a standard $10 \mathrm{~mm}$ full width at half maximum Gaussian kernel before vertex-wise between-group differences and clinical associations were computed.

In order to isolate the effect of occipital cortex atrophy on cognitive performance above that of caudate pathology, manually measured caudate volume was used as a covariate. Caudate atrophy is one of the earliest markers of disease progression, and is sensitive to change in a range of symptoms (Tabrizi et al., 2013, 2012, 2011, 2009). For detailed descriptions of the measurement of caudate volume, see Hobbs et al. (2009). This variable was included as a covariate in regression models fitted between cortical thickness and cognitive task performance.

\subsection{Statistical Analysis}

Summary statistics were calculated for the cognitive and motor tasks, as well as the regional cortical thickness values. Between-groups differences were also examined for these variables (Supplementary material). General linear regression models were used to asses relationships between cognitive task performance (dependent variable) and regional cortical thickness (explanatory variable) in the HD gene-carriers, adjusting for age, gender, study site, education, CAG, disease burden score, HADS depression score and caudate volume. Five models were created for each cognitive task to examine the relationships between performance levels and each occipital region (average occipital cortex, pericalcarine, cuneus, lingual and LOC), resulting in 35 comparisons in total. Semi-partial R-squared (sometimes referred to as eta-squared) was used to determine the unique contribution of 
Running head: OCCIPITAL CORTEX IN HD

occipital cortex thickness to each regression model, i.e., the proportion of variance in each task accounted for by occipital thickness after first controlling for the explanatory power of all other terms in the models.

A FreeSurfer-programmed 'Different Onset, Same Slope' (DOSS) general linear model was fitted to the spatially-normalised thickness data to create statistical maps displaying the relationships between cortical thickness and cognitive task performances in the HD gene-carriers (Fischl and Dale, 2000; Fischl et al., 2004b, 2002). The DOSS model constrains the slopes of any continuous variables to evolve at the same rate in all groups. A DOSS model was deemed appropriate after first investigating that there were no age-related differences on cortical thickness within the groups, the genders or the scanners the models were adjusted for age, gender, study site, education, CAG, disease burden score, HADS depression score and caudate volume. Results were corrected for multiple comparisons using a Monte Carlo cluster-wise simulation with a vertex-wise threshold of $p<0.001$ (twotailed) and a cluster-wise threshold of $p<0.05$, limited to the occipital region (Hagler et al., 2006). A Monte Carlo correction was used as it allows for correction within a particular region, rather than across the whole brain. It uses 10,000 iterations to produce a distribution of no between-group difference under the null hypothesis. From this distribution, the minimum cluster size for threshold-based comparisons is produced (Hagler et al., 2006).

\section{Results}

Participant demographics are detailed in Table 1. The three groups (controls, preHD and HD) were well matched. As expected, the preHD group was younger on average than the other groups $(p<0.05)$. In addition, the HD2 participants showed higher levels of depressive symptoms than other gene positive participants. All cognitive measures and Paced Tapping showed poorer performance with disease progression (Supplementary data).

Figure 1 shows that participants with the HD expanded gene had a thinner average occipital cortex compared with controls. After controlling for age, gender and study site, this reduced thickness in HD gene carriers was significant as early as the preHD-A group ( $>10.8$ years from estimated onset) in the lingual gyrus and the LOC; (all $p<0.05$, Supplementary 
Table 2). In preHD-B individuals ( $<10.8$ years from onset) cortical thickness was significantly lower than in controls for all four occipital regions. All regions showed significantly thinner cortices in the HD1 group compared with the preHD-B group. No significant differences in thickness were found between HD1 and HD2 groups. Statistical maps of between-group differences can be found in the Supplementary Material.

Five of the six cognitive tasks showed statistically significant associations $(p<0.05)$ with averaged occipital cortex thickness, with a thinner cortex associated with poorer performance (Table 2). Mental rotation was the only task that did not correlate with thickness of any region of the occipital lobe. The SDMT showed the strongest association, with average occipital cortex thickness uniquely accounting for an estimated $6.2 \%$ of the variance in task performance. When the four regions of the occipital cortex were examined separately, the LOC and lingual regions were the only regions to show associations with task performance. Thickness of the LOC uniquely explained an estimated $7.5 \%$ of the variance in SDMT performance, followed by 3.4\% for Map Search, 3.0\% for Stroop Word Reading, and $2.9 \%$ for Spot the Change. Thickness of the lingual region showed associations with SDMT (5.0\% of variance uniquely explained), Trails A (4.3\%), Spot the Change performance $(3.1 \%)$ and Map Search (2.2\%). The pericalcarine had one association with SDMT performance, accounting for $1.7 \%$ of the variance in performance. None of the tasks were associated with cuneus thickness, and Paced Tapping task was not associated with the thickness of any region.

The significance maps shown in Figure 2 provide further information on the associations between structure and cognitive performance. SDMT showed a number of large significant clusters spread across the LOC and lingual regions. For all other tasks significant clusters were concentrated on the occipital pole. For Trails A, Spot the Change and Mental Rotation, no significant clusters were detected. 
Running head: OCCIPITAL CORTEX IN HD

Table 1. Demographic data. Data are presented as mean (SD) range.

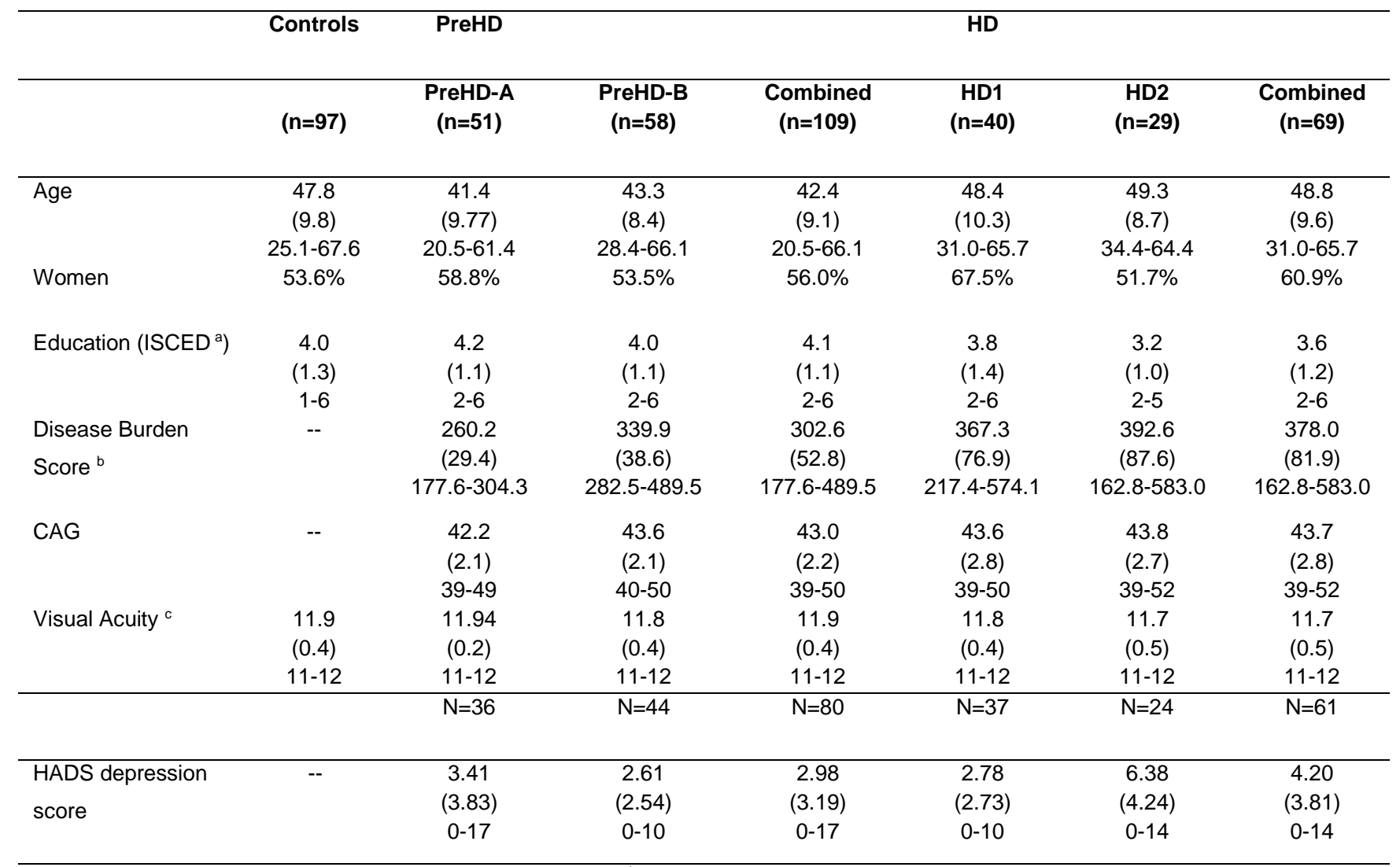

${ }^{\mathrm{a}}$ ISCED = International Standard Classification of Education; ${ }^{\mathrm{b}}$ Disease burden formula (Penney et al., 1997) $=\left(\right.$ CAG-35.5) $\times$ age; ${ }^{\mathrm{c}}$ Snellen visual acuity scale: 1 to 12 represents very poor to high visual acuity respectively.

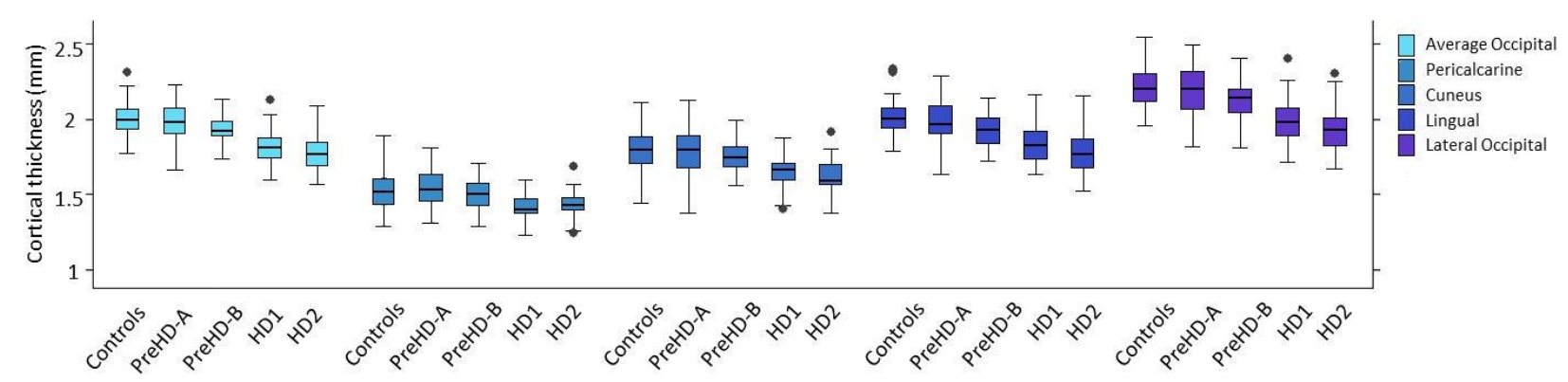

Figure 1. Occipital cortex thickness $(\mathrm{mm})$ plotted by subregion and subgroup. Outliers are shown by circles. 
Table 2: Associations, in the HD gene-carriers, between cortical thickness within occipital regions and cognitive and motor task performance. Light grey shading highlights statistically significant $(p<0.05)$ associations, while dark grey shading represents statistically significant $(p<0.01)$ associations.

\begin{tabular}{|c|c|c|c|c|c|}
\hline & $\begin{array}{c}\text { Average } \\
\text { Occipital } \\
\text { Thickness }\end{array}$ & Pericalcarine & Cuneus & Lingual & LOC \\
\hline Paced Tapping & -95.80 & -21.43 & -37.17 & -70.01 & -87.57 \\
\hline (Deviation from target & $(-210.31,20.15)$ & $(-154.04,111.19)$ & $(-142.12,67.79)$ & $(-173.16,33.14)$ & $(-177.67,2.52)$ \\
\hline interval, & $p=.105$ & $p=.750$ & $\mathrm{p}=.485$ & $p=.182$ & $p=.057$ \\
\hline $\mathrm{N}=141)$ & $\mathrm{R}^{2}=.015$ & $\mathrm{R}^{2}=.000$ & $\mathrm{R}^{2}=.003$ & $\mathrm{R}^{2}=.010$ & $\mathrm{R}^{2}=.021$ \\
\hline SDMT & 32.14 & 19.86 & 10.15 & 25.99 & 27.81 \\
\hline (Number correct, & $(17.34,46.94)$ & $(-1.86,37.85)$ & $(-4.14,24.46)$ & $(12.55,39.43)$ & $(16.40,39.22)$ \\
\hline \multirow[t]{2}{*}{$\mathrm{N}=138)$} & $\mathrm{p}<.001$ & $\mathrm{p}=.031$ & $p=.162$ & $\mathrm{p}<.001$ & $\mathrm{p}<.001$ \\
\hline & $\mathrm{R}^{2}=.062$ & $\mathrm{R}^{2}=.017$ & $\mathrm{R}^{2}=.007$ & $\mathrm{R}^{2}=.050$ & $\mathrm{R}^{2}=.075$ \\
\hline Stroop Word Reading & 29.70 & 15.87 & 12.50 & 17.27 & 26.96 \\
\hline (Number correct, & $(3.44,55.96)$ & $(-15.58,46.15)$ & $(-11.74,36.75)$ & $(-6.53,41.07)$ & $(6.52,47.40)$ \\
\hline \multirow[t]{2}{*}{$N=138)$} & $p=.027$ & $\mathrm{p}=.329$ & $p=.309$ & $p=.154$ & $\mathrm{p}=.010$ \\
\hline & $\mathrm{R}^{2}=.022$ & $R^{2}=.004$ & $\mathrm{R}^{2}=.005$ & $\mathrm{R}^{2}=.010$ & $R^{2}=.030$ \\
\hline Trails A & -19.32 & -16.77 & -5.61 & -23.41 & -13.00 \\
\hline (Time in seconds, & $(-36.33,-2.31)$ & $(-36.62,3.08)$ & $(-21.35,10.13)$ & $(-38.40,-8.43)$ & $(-26.40, .40)$ \\
\hline \multirow[t]{2}{*}{$N=138)$} & $p=.026$ & $\mathrm{p}=.097$ & $\mathrm{p}=.482$ & $\mathrm{p}=.002$ & $\mathrm{p}=.057$ \\
\hline & $\mathrm{R}^{2}=.023$ & $\mathrm{R}^{2}=.013$ & $\mathrm{R}^{2}=.002$ & $R^{2}=.043$ & $\mathrm{R}^{2}=.017$ \\
\hline Map search & 23.02 & 5.39 & 3.89 & 19.72 & 21.39 \\
\hline (Number correct, & $(3.73,42.31)$ & $(-17.69,28.48)$ & $(-14.16,21.93)$ & $(2.61,36.84)$ & $(6.49,36.30)$ \\
\hline \multirow[t]{2}{*}{$N=135)$} & $p=.020$ & $p=.645$ & $p=.671$ & $p=.024$ & $\mathrm{p}=.005$ \\
\hline & $R^{2}=.024$ & $\mathrm{R}^{2}=.001$ & $\mathrm{R}^{2}=.001$ & $\mathrm{R}^{2}=.022$ & $\mathrm{R}^{2}=.034$ \\
\hline Mental Rotation & .08 & .05 & -.01 & .04 & .10 \\
\hline (Percent correct, & $(-.11, .28)$ & $(-.17,28)$ & $(-.19, .16)$ & $(-.14, .21)$ & $(-.06, .24)$ \\
\hline \multirow[t]{2}{*}{$N=138)$} & $p=.392$ & $\mathrm{p}=.643$ & $\mathrm{p}=.869$ & $\mathrm{p}=.654$ & $p=.238$ \\
\hline & $\mathrm{R}^{2}=.004$ & $R^{2}=.001$ & $R^{2}=.000$ & $\mathrm{R}^{2}=.001$ & $\mathrm{R}^{2}=.007$ \\
\hline Spot the Change & 18.18 & 9.47 & -.78 & 18.16 & 15.43 \\
\hline (Percent correct, & $(.89,35.47)$ & $(-10.50,29.43)$ & $(-16.66,15.10)$ & $(2.80,33.53)$ & $(1.90,28.97)$ \\
\hline \multirow[t]{2}{*}{$N=141)$} & $p=.040$ & $\mathrm{p}=.350$ & $\mathrm{p}=.923$ & $p=.021$ & $p=.026$ \\
\hline & $\mathrm{R}^{2}=.024$ & $\mathrm{R}^{2}=.005$ & $\mathrm{R}^{2}=.000$ & $\mathrm{R}^{2}=.031$ & $\mathrm{R}^{2}=.029$ \\
\hline
\end{tabular}




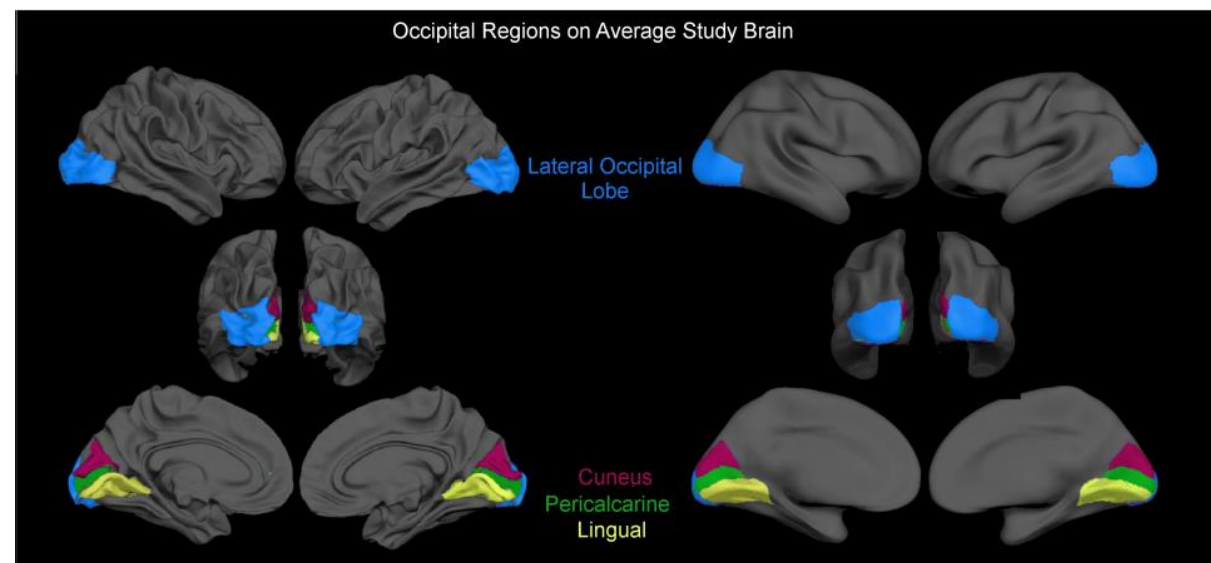

Symbol Digit Modalities Test

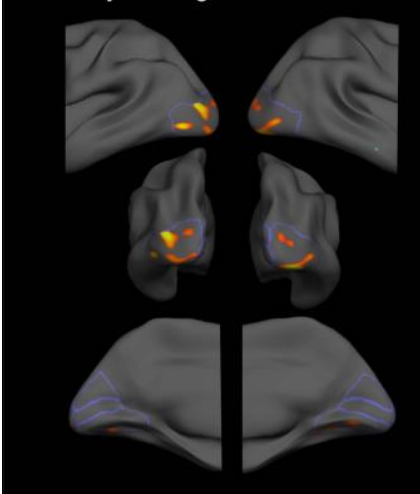

Trails A

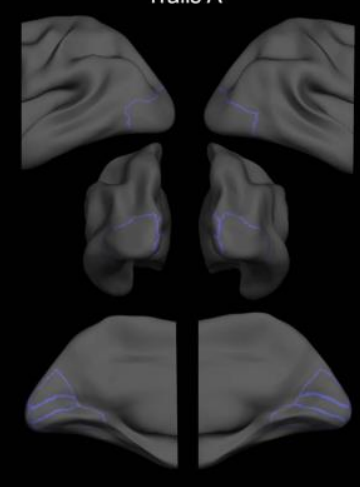

Left Hemisphere
Stroop Word Reading

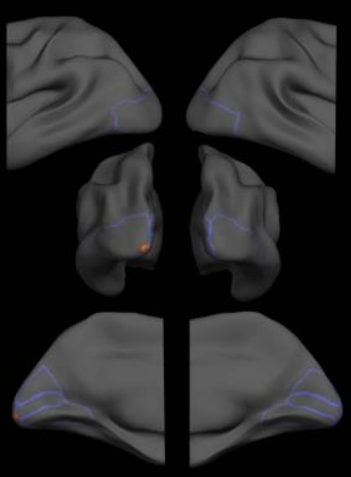

Spot the Change

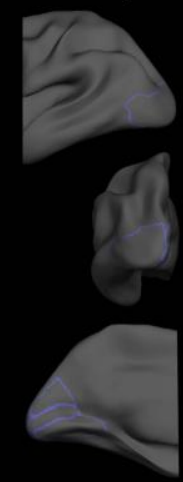

$\begin{array}{llll}0.0001 & 0.05 & 0.05 & 0.0001\end{array}$

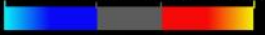

Map Search

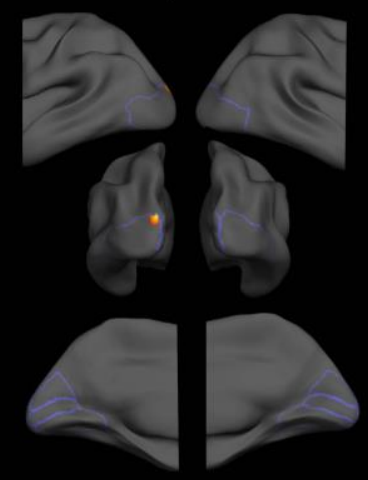

Mental rotation

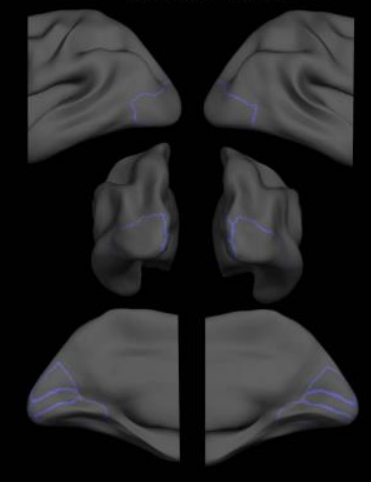

Right Hemisphere

Figure 2. A: The Lateral occipital, cuneus, lingual and pericalcarine atlas regions as defined by the Desikan-Killiany atlas overlaid on the study-specific average cortical template and on the inflated study-specific average cortical template. B: Significance maps of the associations $(0.0001<p<0.05)$ between occipital cortex thickness and cognitive task performance in the HD gene-carriers, displayed on inflated brain templates. Associations were adjusted for age, gender, study site, education, CAG, disease burden score, HADS depression score and caudate volume, and corrected for multiple comparisons using Monte Carlo cluster-wise correction $(p<0.05)$ across the four occipital regions. 
Running head: OCCIPITAL CORTEX IN HD

\section{Discussion}

This study is the first to show a significant, but heterogeneous, effect of disease stage on cortical thickness of the occipital lobe. The greatest group differences in cortical thickness were found in the LOC and the lingual regions, and these differences were apparent more than 10 years from disease onset. We also demonstrate, for the first time, significant regionally-specific associations between occipital cortical thickness and cognitive task performance in an HD cohort with normal visual acuity. The associations were significant after controlling for a number of factors, including caudate volume and disease burden, and were most notable within the LOC and the lingual gyrus. These two occipital regions are known to be involved in higher-level visual functioning such as word reading, visual memory, integration of visual and tactile information and object recognition. In contrast, the pericalcarine gyrus showed one significant correlation with cognition, and cuneus did not show any significant associations with cognitive task performance.

Performance on all cognitive tasks and Paced Tapping was poorer in participants with more advanced disease status (Supplementary tables 1 and 2). Consistent with previously published findings, HD gene-carriers had thinner occipital cortices both prior to disease onset and in manifest disease, compared with healthy controls (Rosas et al., 2008; Tabrizi et al., 2009). The between-group differences were heterogeneous across the four occipital regions; differences in thickness of the lingual and LOC regions, which are associated with more heteromodal, high level visual processing, were affected earliest and most severely, whereas regions associated with more unimodal basic visual processing activities, including the cuneus and the pericalcarine, were less affected and emerged only in the groups with diagnosed disease (HD1, HD2). In addition, the greatest between-group difference appeared between HD gene-carriers nearing onset and those in the early stages of disease (i.e. preHD-B vs. HD1), which suggests a possible increase in the rate of occipital cortex thinning around disease onset, although this should be examined in a longitudinal analysis. After disease onset, atrophy appeared to plateau somewhat, with no significant difference in any region between gene-carriers with a TFC of 11-13 (HD stage 1) and those with a TFC of 7- 
Running head: OCCIPITAL CORTEX IN HD

10 (HD stage 2). These results extend upon previous findings by providing a detailed characterization on both the location and the progression of occipital lobe atrophy in HD.

Combined across all regions, occipital cortex thickness in HD gene-carriers was associated with performance on five of the six cognitive tasks, all of which included a visual component. When examining the sub-regions, thickness of the pericalcarine correlated with one cognitive task, SDMT, although the effect size was small $\left(\mathrm{R}^{2}=0.017\right)$. The pericalcarine is the initial region of visual processing; $V 1$ maps the signals received from the retina in a topographic manner (Tootell et al., 1998; Wandell et al., 2007). While V1 does have cells specialised for functions such as orientation detection and motion selection, it only performs basic filtering processes before sending the information onto other regions for more advanced processing. The cuneus was not associated with performance on any cognitive task, and this region is also thought to process basic visual properties, such as colour, motion detection and orientation information. The results indicate that thinning of two occipital regions performing the most basic visual processes has a very limited effect on cognitive performance. Alternately, there may be effects of thinning in these basic visual processing regions that we did not detect using the cognitive tasks in our battery.

The LOC and lingual regions showed significant relationships with performance on a number of cognitive tests. After controlling for caudate volume, the thickness of the LOC was estimated to account for between $2.9 \%$ and $7.5 \%$ of variance in performance on the cognitive tasks, and lingual thickness was estimated to account for $2.2 \%$ to $5.0 \%$ of performance variance. The LOC is a large region known to have a role in complex visual processing, with object recognition and integration of visual information two of the many functions performed by the LOC (Beauchamp, 2005; Grill-Spector et al., 2001; Larsson and Heeger, 2006). The lingual region is known to process detailed colour information (area V4) and visual memory. Mental rotation was the only task that was not associated with any region. This result might reflect an insensitivity of the selected outcome measure, percent correct. While previous studies have shown associations between cognitive performance and occipital lobe atrophy (Gomez-Tortosa et al., 1996; Rosas et al., 2005; Scahill et al., 
2013). The use of a regional analysis that carefully controls for both a number of important disease-related factors and for multiple comparisons has not previously been used. The results provide evidence that distinct regions of the occipital lobe play a vital part in cognitive performance and that task performance is impacted by occipital lobe pathology.

Furthermore, regions associated with higher-level complex visual processing appear to play a greater role in performance than areas involved in basic visual processes. No significant associations were found between Paced Tapping and thickness of any region of the occipital lobe. As this motor task is a significant predictor of disease progression in HD (Tabrizi et al., 2013), the lack of significant associations suggest that the impact of occipital thickness on these cognitive tasks are specific to visual cognition rather than general disease progression.

Cognitive deficits in diseases such as HD, Alzheimer's disease, schizophrenia and bipolar disorder (Holroyd et al., 2000; Lyoo et al., 2006), in which occipital atrophy is present in the absence of reported visual deficits, should be interpreted with the current results in mind; it is possible that the reduced cognitive performance seen in these diseases could be in part due to impairments in higher-level visual processing, rather than just problems with executive functioning and cognition. Furthermore, this study has implications for the interpretation of cognitive deficits in a clinical setting. Another neurodegenerative condition, posterior cortical atrophy, is characterized by extensive atrophy in the posterior and occipital regions of the brain which is reflected in visuospatial and visuoperceptual impairments (Crutch et al., 2012). Patients are encouraged to use adapted visual aids (i.e., mobile phones with clearer display settings, improved lighting) to reduce the effects of this condition on everyday life. This management technique may be relevant for other conditions, including $\mathrm{HD}$, when occipital atrophy is present and appears to be having some effect on cognitive performance.

The study has several limitations. Firstly, we cannot establish causality between cortical thickness and cognitive performance from our data; techniques such as functional MRI may provide more direct evidence of a link between cognitive impairment and neuronal 
function, and methodologies for interrogating large multi-site fMRI datasets are in the process of validation. Nevertheless, previous work has demonstrated the strong predictive power of atrophy measures in disease progression (Tabrizi et al., 2013) and it seems likely that this structural damage may underlie much of the functional impairment seen in HD gene carriers. In addition, while some studies have investigated aspects of basic visual processing in HD (O'Donnell et al., 2008, 2003), more work is needed with tasks specifically designed to probe visual processing to firmly establish elements of visual processing that are affected by cortical thickness. The lack of significant difference in cortical thickness in HD1 compared to HD2 may be a result of low power rather than a biological slowing of atrophy at this point in the disease. Similarly, the non-significant, biologically implausible thicker cortex in the pericalcarine region found in HD2 compared to HD1 groups could be an artefact of the segmentation technique, as the pericalcarine is a region buried deep within a sulcus and thus the measurement is likely to include some noise. This result is currently being examined in a larger study of atrophy measures. Another limitation that should be considered is that there may be an effect of medication use on cognitive performance. Many HD patients are on some form of medication (e.g. antidepressants, sleeping aids). However, due to the wide range of medications and dosing levels, it is difficult to account for effectively in analyses and for that reason we have not controlled for medication as a factor in this study. Finally, similar structure-function associations between neural change and cognitive performance have been observed in Alzheimer's disease, schizophrenia and bipolar disorder (Holroyd et al., 2000; Lyoo et al., 2006). Occipital atrophy is also present in these conditions in the absence of overt visual deficits, although the specific pattern of atrophy and time of onset present in $\mathrm{HD}$ is likely to differ from that of other diseases. Due to these differences, further investigation of the relationships in these clinical cohorts is warranted before reaching conclusions on the applicability of the current findings.

The significant associations between occipital cortex thickness and cognitive task performance demonstrate that while reduced occipital cortex thickness may not manifest itself in patient reported visual deficits, there appears to be a substantial impact on higher- 
Running head: OCCIPITAL CORTEX IN HD

level visual functioning and consequently cognitive task performance. The visual cortex makes a significant yet subtle contribution to cognitive task performance beyond that of other disease related factors. This contribution should be considered during the interpretation of cognitive task performance and potentially in clinical care for both HD and other conditions in which occipital atrophy occurs. 
Running head: OCCIPITAL CORTEX IN HD

\section{Funding}

This work was supported by the $\mathrm{CHDI} /$ High Q Foundation, a not for profit organisation dedicated to finding treatments for Huntington's disease.

\section{Acknowledgements}

We wish to thank all those at the CHDI /High Q Foundation responsible for the TRACK-HD study, in particular Beth Borowsky, Allan Tobin, Daniel van Kammen, Ethan Signer and Sherry Lifer. The authors also wish to extend their gratitude to the TRACK-HD study participants and families. This work was undertaken at UCLH/UCL which received a proportion of funding from the Department of Health's NIHR Biomedical Research Centres funding scheme. SJT acknowledges support of the National Institute for Health Research through the Dementias and Neurodegenerative Research Network, DeNDRoN.

\section{The TRACK-HD Investigators:}

Eric Axelson, lowa; Natalie Bechtel, Munster; Simon J.A. van Bogaard, Leiden; Stefan Bohlen, Munster; Jenny Callaghan, Manchester; Colin Campbell and Melissa Campbell, Monash; David Cash, IXICO; Allison Coleman, Vancouver; David Craufurd, Manchester; Rachelle Dar Santos and Joji Decolongon, Vancouver; Eve Dumas, Leiden; Nick Fox, UCL; Chris Frost, LSHTM; Jeroen van der Grond and Ellen t'Hart, Leiden; Stephen Hicks, Oxford; Nicola Hobbs, UCL; Céline Jauffret, Paris; Hans Johnson, lowa; Rebecca Jones, LSHTM; Damian Justo, Paris; Chris Kennard, Oxford; Nayana Lahiri, UCL; Bernhard Landwehmeyer, Ulm; Stephane Lehericy, Paris; Ian Malone, UCL; Cecilia Marelli, Paris; Cassie Milchman, Monash; Kevin Nigaud, Paris; Gail Owen, UCL; Tracey Pepple, UCL; Sarah Queller, Indiana; Joy Read and Miranda Say, UCL; Aaron Sturrock, Vancouver; Romain Valabrègue, Paris;

Sturrock, Vancouver; Romain Valabrègue, Paris; Chiachi Wang, lowa. 


\section{References}

medi, A., 2002. Convergence of visual and tactile shape processing in the human lateral occipital complex. Cereb Cortex 12, 1202-1212. doi:10.1093/cercor/12.11.1202

Astafiev, S. V, Stanley, C.M., Shulman, G.L., Corbetta, M., 2004. Extrastriate body area in human occipital cortex responds to the performance of motor actions. Nat Neurosci 7, 542-8. doi:10.1038/nn1241

Balcer, L.J., Baier, M.L., Pelak, V.S., Fox, R.J., Shuwairi, S., Galetta, S.L., Cutter, G.R., Maguire, M.G., 2000. New low-contrast vision charts: reliability and test characteristics in patients with multiple sclerosis. Mult.Scler. 6, 163-171.

Beauchamp, M.S., 2005. See me, hear me touch me: multisensory intergration in lateral occipital-temporal cortex. Curr Opin Neurobiol 15, 145-153.

Boyes, R.G., Gunter, J.L., Frost, C., Janke, A.L., Yeatman, T., Hill, D.L.G., Bernstein, M.A., Thompson, P.M., Weiner, M.W., Schuff, N., Alexander, G.E., Killiany, R.J., DeCarli, C., Jack, C.R., Fox, N.C., 2008. Intensity non-uniformity correction using N3 on 3-T scanners with multichannel phased array coils. Neuroimage 39, 1752-62. doi:10.1016/j.neuroimage.2007.10.026

Cabeza, R., Nyberg, L., 2000. Imaging cognition II: an empirical review of 275 PET and fMRI studies. J Cogn Neurosci 12, 1-47.

Cowan, N., Elliott, E.M., Scott, S.J., Morey, C.C., Mattox, S., Hismjatullina, A., Conway, A.R., 2005. On the capacity of attention: its estimation and its role in working memory and cognitive aptitudes. Cogn Psychol 51, 42-100.

Crutch, S.J., Lehmann, M., Schott, J.M., Rabinovici, G.D., Rossor, M.N., Fox, N.C., 2012. Posterior cortical atrophy. Lancet Neurol 11, 170-8. doi:10.1016/S14744422(11)70289-7

De Souza, J., Jones, L.A., Rickards, H., 2010. Validation of self-report depression rating scales in Huntington's disease. Mov Disord 25, 91-96. doi:10.1002/mds.22837

Desikan, R.S., Segonne, F., Fischl, B., Quinn, B.T., Dickerson, B.C., Blacker, D., Buckner, R.L., Dale, A.M., Maguire, R.P., Hyman, B.T., Albert, M.S., Killiany, R.J., 2006. An automated labeling system for subdividing the human cerebral cortex on MRI scans into gyral based regions of interest. Neuroimage 31, 968-980.

Fischl, B., Dale, A.M., 2000. Measuring the thickness of the human cerebral cortex from magnetic resonance images. Proc.Natl.Acad.Sci.U.S.A 97, 11050-11055.

Fischl, B., Salat, D.H., Busa, E., Albert, M., Dieterich, M., Haselgrove, C., van der Kouwe, A., Killiany, R., Kennedy, D., Klaveness, S., Montillo, A., Makris, N., Rosen, B., Dale, A.M., 2002. Whole brain segmentation: automated labeling of neuroanatomical structures in the human brain. Neuron 33, 341-355.

Fischl, B., Salat, D.H., van der Kouwe, A.J., Makris, N., Segonne, F., Quinn, B.T., Dale, A.M., 2004a. Sequence-independent segmentation of magnetic resonance images. Neuroimage 23, S69-S84. 
Fischl, B., van der Kouwe, A., Destrieux, C., Halgren, E., Segonne, F., Salat, D.H., Busa, E., Seidman, L.J., Goldstein, J., Kennedy, D., Caviness, V., Makris, N., Rosen, B., Dale, A.M., 2004b. Automatically parcellating the human cerebral cortex. Cereb Cortex 14, $11-22$.

Gegenfurtner, K.R., Kiper, D.C., Levitt, J.B., 1997. Functional properties of neurons in macaque area V3. J Neurophysiol 77, 1906-1923.

Gomez-Anson, B., Alegret, M., Munoz, E., Monte, G.C., Alayrach, E., Sanchez, A., Boada, M., Tolosa, E., 2009. Prefrontal cortex volume reduction on MRI in preclinical Huntington's disease relates to visuomotor performance and CAG number. Park Disord 15, 213-219.

Gomez-Tortosa, E., del Barrio, A., Barroso, T., Garcia Ruiz, P.J., 1996. Visual processing disorders in patients with Huntington's disease and asymptomatic carriers. J Neurol 243, 286-292.

Grill-Spector, K., Kourtzi, Z., Kanwisher, N., 2001. The lateral occipital complex and its role in object recognition. Vis Res 41, 1409-1422.

Hagler, D.J., Saygin, A.P., Sereno, M.I., 2006. Smoothing and cluster thresholding for cortical surface-based group analysis of fMRI data. Neuroimage 33, 1093-103. doi:10.1016/j.neuroimage.2006.07.036

Hebart, M.N., Hesselmann, G., 2012. What visual information is processed in the human dorsal stream? J Neurosci 32, 8107-9. doi:10.1523/JNEUROSCI.1462-12.2012

Hobbs, N.Z., Henley, S.M., Wild, E.J., Leung, K.K., Frost, C., Barker, R.A., Scahill, R.I., Barnes, J., Tabrizi, S.J., Fox, N.C., 2009. Automated quantification of caudate atrophy by local registration of serial MRI: evaluation and application in Huntington's disease. Neuroimage 47, 1659-1665.

Holroyd, S., Shepherd, M.L., Downs, J.H.I., 2000. Occipital atrophy is associated with visual hallucinations in Alzheimer's disease. J Neuropsychiatry ClinNeurosci 12, 25-28.

Huntington Study Group, 1996. Unified Huntington's Disease Rating Scale: reliability and consistency. Mov Disord 11, 136-142.

Langbehn, D.R., Hayden, M.R., Paulsen, J.S., 2010. CAG-repeat length and the age of onset in Huntington disease (HD): a review and validation study of statistical approaches. AmJ MedGenetB NeuropsychiatrGenet 153B, 397-408.

Lange, H., 1981. Quantitative changes of telencephalon, diencephalon, and mesencephalon in Huntington's chorea, postencephalitic, and idiopathic parkinsonism. Verh Anat Ges $75,923-925$.

Larsson, J., Heeger, D.J., 2006. Two retinotopic visual areas in human lateral occipital cortex. J Neurosci 26, 13128-13142.

Lyoo, I.K., Sung, Y.H., Dager, S.R., Friedman, S.D., Lee, J.Y., Kim, S.J., Kim, N., Dunner, D.L., Renshaw, P.F., 2006. Regional cerebral cortical thinning in bipolar disorder. Bipolar.Disord. 8, 65-74. 
MacLeod, C.M., 1991. Half a century of research on the Stroop effect: an integrative review. Psychol.Bull. 109, 163-203.

Mechelli, A., Humphreys, G.W., Mayall, K., Olson, A., Price, C.J., 2000. Differential effects of word length and visual contrast in the fusiform and lingual gyri during reading. Proc.Biol.Sci. 267, 1909-1913.

O'Donnell, B.F., Blekher, T.M., Weaver, M., White, K.M., Marshall, J., Beristain, X., Stout, J.C., Gray, J., Wojcieszek, J.M., Foroud, T.M., 2008. Visual perception in prediagnostic and early stage Huntington's disease. J Int Neuropsychol Soc 14, 446-53. doi:10.1017/S1355617708080405

O’Donnell, B.F., Wilt, M.A., Hake, A.M., Stout, J.C., Kirkwood, S.C., Foroud, T., 2003. Visual function in Huntington's disease patients and presymptomatic gene carriers. Mov Disord 18, 1027-34. doi:10.1002/mds.10491

Paulsen, J.S., Langbehn, D.R., Stout, J.C., Aylward, E.H., Ross, C.A., Nance, M.A., Guttman, M., Johnson, S., MacDonald, M., Beglinger, L.J., Duff, K., Kayson, E., Biglan, K., Shoulson, I., Oakes, D., Hayden, M., 2008. Detection of Huntington's disease decades before diagnosis: the Predict-HD study. J Neurol Neurosurg Psychiatry 79, 874-880.

Paulsen, J.S., Long, J.D., Ross, C.A., Harrington, D.L., Erwin, C.J., Williams, J.K., Westervelt, H.J., Johnson, H.J., Aylward, E.H., Zhang, Y., Bockholt, H.J., Barker, R.A., 2014. Prediction of manifest Huntington's disease with clinical and imaging measures: a prospective observational study. Lancet Neurol 13, 1193-1201. doi:10.1016/S14744422(14)70238-8

Penney, J.B., Vonsattel, J.P., MacDonald, M.E., Gusella, J.F., Myers, R.H., 1997. CAG repeat number governs the development rate of pathology in Huntington's disease. Ann Neurol 41, 689-92. doi:10.1002/ana.410410521

Reilmann, R., Kirsten, F., Bohlen, S., Saemann, P., Merl, T., Auer, D., 2005. Multimodal objective assessment of motor deficits in Huntington's disease using isometric force analysis. J neurolNeurosurg Psychiatry 76.

Robertson, I.H., Ward, T., Ridgeway, V., Nimmo-Smith, I., 1994. The Test of Everyday Attention. Thames Valley Test Co, Bury St. Edmunds (UK).

Roe, A.W., Ts'o, D.Y., 1995. Visual topography in primate V2: multiple representation across functional stripes. J Neurosci 15, 3689-3715.

Rosas, H.D., Hevelone, N.D., Zaleta, A.K., Greve, D.N., Salat, D.H., Fischl, B., 2005. Regional cortical thinning in preclinical Huntington disease and its relationship to cognition. Neurology 65, 745-747.

Rosas, H.D., Liu, A.K., Hersch, S., Glessner, M., Ferrante, R.J., Salat, D.H., van der Kouwe, A., Jenkins, B.G., Dale, A.M., Fischl, B., 2002. Regional and progressive thinning of the cortical ribbon in Huntington's disease. Neurology 58, 695-701.

Rosas, H.D., Salat, D.H., Lee, S.Y., Zaleta, A.K., Pappu, V., Fischl, B., Greve, D., Hevelone, N., Hersch, S.M., 2008. Cerebral cortex and the clinical expression of Huntington's 
disease: complexity and heterogeneity. Brain 131, 1057-1068.

Rüb, U., Seidel, K., Vonsattel, J.P., Lange, H.W., Eisenmenger, W., Götz, M., Del Turco, D., Bouzrou, M., Korf, H.-W., Heinsen, H., 2015. Huntington's disease (HD): neurodegeneration of Brodmann's Primary Visual Area 17 (BA17). Brain Pathol. doi:10.1111/bpa.12237

Scahill, R.I., Hobbs, N.Z., Say, M.J., Bechtel, N., Henley, S.M.D., Hyare, H., Langbehn, D.R., Jones, R., Leavitt, B.R., Roos, R. a C., Durr, A., Johnson, H., Lehéricy, S., Craufurd, D., Kennard, C., Hicks, S.L., Stout, J.C., Reilmann, R., Tabrizi, S.J., 2013. Clinical impairment in premanifest and early Huntington's disease is associated with regionally specific atrophy. Hum Brain Mapp 34, 519-529. doi:10.1002/hbm.21449

Shepard, R., Metzler, J., 1971. Mental rotation of three dimensional objects. Science (80- ) $171,701-703$.

Sled, J.G., Zijdenbos, A.P., Evans, A.C., 1998. A nonparametric method for automatic correction of intensity nonuniformity in MRI data. IEEE TransMedlmaging 17, 87-97. doi:10.1109/42.668698

Smith, A., 1991. Symbol Digit Modalities Test . Western Psychological Services, Los Angeles.

Spreen, O., Strauss, E., 1985. A compendium of neuropsychological tests: Administration, norms and commentary . Oxford University Press, New York.

Stoffers, D., Sheldon, S., Kuperman, J.M., Goldstein, J., Corey-Bloom, J., Aron, A.R., 2010. Contrasting gray and white matter changes in preclinical Huntington disease: an MRI study. Neurology 74, 1208-1216.

Tabrizi, S.J., Langbehn, D.R., Leavitt, B.R., Roos, R.A., Durr, A., Craufurd, D., Kennard, C., Hicks, S.L., Fox, N.C., Scahill, R.I., Borowsky, B., Tobin, A.J., Rosas, H.D., Johnson, H., Reilmann, R., Landwehrmeyer, B., Stout, J.C., 2009. Biological and clinical manifestations of Huntington's disease in the longitudinal TRACK-HD study: crosssectional analysis of baseline data. Lancet Neurol 8, 791-801. doi:10.1016/S14744422(09)70170-X

Tabrizi, S.J., Reilmann, R., Roos, R., Durr, A., Leavitt, B., Owen, G., Jones, R., Johnson, H., Craufurd, D., Hicks, S., Kennard, C., Landwehrmeyer, B., Stout, J.C., Borowsky, B., Scahill, R.I., Frost, C., Langbehn, D.R., 2012. Potential endpoints for clinical trials in premanifest and early Huntington's disease in the TRACK-HD study: analysis of 24 month observational data. Lancet Neurol 11, 42-53. doi:10.1016/S14744422(11)70263-0

Tabrizi, S.J., Scahill, R.I., Durr, A., Roos, R.A., Leavitt, B.R., Jones, R., Landwehrmeyer, G.B., Fox, N.C., Johnson, H., Hicks, S.L., Kennard, C., Craufurd, D., Frost, C., Langbehn, D.R., Reilmann, R., Stout, J.C., 2011. Biological and clinical changes in premanifest and early stage Huntington's disease in the TRACK-HD study: the 12month longitudinal analysis. Lancet Neurol 10, 31-42.

Tabrizi, S.J., Scahill, R.I., Owen, G., Durr, A., Leavitt, B.R., Roos, R.A., Borowsky, B., Landwehrmeyer, B., Frost, C., Johnson, H., Craufurd, D., Reilmann, R., Stout, J.C., 
Langbehn, D.R., 2013. Predictors of phenotypic progression and disease onset in premanifest and early-stage Huntington's disease in the TRACK-HD study: analysis of 36-month observational data. Lancet Neurol 12, 637-649.

Tootell, R.B., Hadjikhani, N.K., Vanduffel, W., Liu, A.K., Mendola, J.D., Sereno, M.I., Dale, A.M., 1998. Functional analysis of primary visual cortex (V1) in humans.

Proc.Natl.Acad.Sci.U.S.A 95, 811-817.

Tootell, R.B., Tsao, D., Vanduffel, W., 2003. Neuroimaging weighs in: humans meet macaques in "primate" visual cortex. J Neurosci 23, 3981-3989.

Vonsattel, J.P., DiFiglia, M., 1998. Huntington disease. J NeuropatholExpNeurol 57, 369384.

Wandell, B.A., Dumoulin, S.O., Brewer, A.A., 2007. Visual field maps in human cortex. Neuron 56, 366-383.

Wolf, R.C., Sambataro, F., Vasic, N., Baldas, E.M., Ratheiser, I., Bernhard, L.G., Depping, M.S., Thomann, P.A., Sprengelmeyer, R., Sussmuth, S.D., Orth, M., 2014. Visual system integrity and cognition in early Huntington's disease. Eur.J Neurosci. 40, 24172426.

Zeki, S.M., 1978. Functional specialisation in the visual cortex of the rhesus monkey. Nature 274, 423-428.

Zigmond, A.S., Snaith, R.P., 1983. The Hospital Anxiety and Depression Scale. Acta Psychiatr Scand 67, 361-370. doi:10.1111/j.1600-0447.1983.tb09716.x 